\title{
Relação entre determinantes socioeconômicos e hábitos bucais de risco para más-oclusões em pré-escolares ${ }^{\dagger}$
}

\section{The relationship between socioeconomic determinants and oral habits as risk factors for malocclusion in preschool children}

\author{
Nilce Emy TOMITA* \\ Aubrey SHEIHAM** \\ Vitoriano Truvijo BIJELLA*** \\ Laércio Joel FRANCO****
}

\begin{abstract}
TOMITA, N. E.; SHEIHAM, A.; BIJELLA, V. T.; FRANCO, L. J. Relação entre determinantes socioeconômicos e hábitos bucais de risco para más-oclusões em pré-escolares. Pesq Odont Bras, v. 14, n. 2, p. 169-175, abr./jun. 2000.

Tendo por objetivo avaliar como determinantes socioeconômicos afetam a prevalência de hábitos bucais deletérios em pré-escolares, este estudo transversal foi desenvolvido. O inquérito epidemiológico foi realizado no período de outubro de 1994 a dezembro de 1995. A amostra probabilística foi constituída por 2.139 crianças, de ambos os sexos, na faixa etária de 3 a 5 anos, matriculadas em instituições públicas ou privadas do município de Bauru - SP - Brasil. Uma subamostra de 618 crianças apresentou resposta ao questionário socioeconômico. A partir da hipótese que determinantes socioeconômicos afetam o estado emocional da criança e isto se manifesta através de hábitos bucais, como sucção de chupeta e sucção digital, foram realizadas análises bivariadas envolvendo as respostas ao questionário socieconômico e algumas variáveis de exposição. Alguns determinantes socioeconômicos, como o trabalho materno e ocupação da pessoa de maior renda no domicílio estão relacionados com a maior prevalência de hábitos bucais $(\mathrm{p}<0,05)$, que por sua vez estão positivamente associados com a má-oclusão.
\end{abstract}

UNITERMOS: Epidemiologia; Maloclusão; Fatores socioeconômicos; Hábitos bucais.

\section{INTRODUÇÃO}

A má-oclusão constitui uma anomalia do desenvolvimento dos dentes e/ou arcos dentários, ocasionando de desconforto estético, nos casos mais leves, a agravos funcionais e incapacitações, nos casos mais severos ${ }^{6}$.

A prevenção da má-oclusão é considerada uma alternativa potencial ao tratamento, uma vez que as más-oclusões mais comuns são condições funcionais adquiridas, atribuídas a dietas pastosas, problemas respiratórios e hábitos bucais deleté$\operatorname{rios}^{29}$.

Os hábitos bucais, por sua vez, podem ser influenciados, assim como outros comportamentos, por alguns fatores sociais, como emprego da mãe, inicialmente pelo padrão de aleitamento, tempo que a criança permanece na escola (periodo inte- gral ou parcial), renda familiar, algumas doenças respiratórias e problemas de fala, entre outros ${ }^{13}$.

Adicionalmente, alguns estudos têm demonstrado que a condição socioeconômica apresenta influência sobre o peso ao nascer ${ }^{2,8}$, doenças respiratórias $^{8}$, aleitamento materno ${ }^{8,19}$ e dificuldades de acesso aos serviços odontológicos ${ }^{6}$. Estas variáveis devem ser melhor avaliadas, de forma a estabelecer como os fatores socioeconômicos podem influenciar a má-oclusão através de hábitos bucais, fatores psicológicos e padrões de doenças gerais. Um modelo teórico é proposto ${ }^{29}$, levando-se em consideração estes fatores (Figura 1).

É interessante observar que a maior parte dos estudos realizados com populações em idade pré-escolar reafirma a influência deletéria da persistência dos hábitos bucais após os 3 anos de idade sobre a oclusão (Quadro 1). A etiologia dos hábi-

\footnotetext{
† Tese para Obtenção do Título de Doutor em Odontologia na área de Odontopediatria.

* Professora Doutora; *** Professor Titular - Departamento de Odontopediatria, Ortodontia e Saúde Coletiva da Faculdade de Odontologia de Bauru da USP.

** Professor, Department of Epidemiology and Public Health, University College London Medical School.

**** Professor Titular do Departamento de Medicina Preventiva da Escola Paulista de Medicina da UNIFESP.
} 
TOMITA, N. E.; SHEIHAM, A.; BIJELLA, V. T.; FRANCO, L. J. Relação entre determinantes socioeconômicos e hábitos bucais de risco para más-oclusões em pré-escolares. Pesq Odont Bras, v. 14, n. 2, p. 169-175, abr./jun. 2000.

tos bucais, entretanto, tem recebido escassa atenção.

A hipótese de trabalho é que os fatores socioeconômicos afetam o estado psicológico da criança. Isto se manifesta através de hábitos bucais, como sucção de chupeta e sucção digital e afetam a condição oclusal.

O presente estudo tem por objetivos avaliar a relação entre algumas condições socioeconômicas e fatores ambientais que influenciam a prevalência de hábitos bucais deletérios e a má-oclusão em pré-escolares.

\section{CASUÍSTICA E MÉTODOS}

A população-alvo foi constituída por crianças em idade pré-escolar, de ambos os sexos, matriculadas em instituições de ensino, públicas ou privadas, da zona urbana do município de Bauru - SP. A faixa etária escolhida foi a dos 3 aos 5 anos, devido à cronologia de erupção dentária ${ }^{12}$.

\section{Identificação da população-alvo}

Em fevereiro de 1994, solicitou-se às instituições as listagens de crianças matriculadas, com as respectivas datas de nascimento. O objetivo foi conhecer o universo de crianças presentes nas quatro categorias de instituições: creche municipal, creche particular, escola particular e escola municipal de educação infantil (EMEI). Das 95 instituições do município, 49 responderam à solicitação. A relação de crianças matriculadas totalizou 4.858. Considerando-se o número médio de alunos por instituição em 99,1 (4.858/49) estimou-se em $9.418(\mathrm{~N})$ o número de crianças matriculadas na totalidade das instituições pré-escolares do município.

O tamanho da amostra foi calculado segundo a fórmula de $\mathrm{COCHRAN}^{7}$ :

$$
n=\frac{\frac{t_{\frac{\alpha}{2}}^{2} p(1-p)}{d^{2}}}{1+\frac{1}{N}\left(\frac{t_{\frac{a}{2}}^{2} p(1-p)}{d^{2}}-1\right)}
$$

adotando-se $p=0,8$ (estimativa da prevalência de má-oclusão), intervalo de confiança de $1-\alpha=0,95$, erro amostral $d=1,5$ e $N=9.418$, estimou-se uma amostra com 2.117 crianças, como adequada para as análises propostas. Finalmente, prevendo-se perdas, foi sorteado um excesso de 40,0\% na

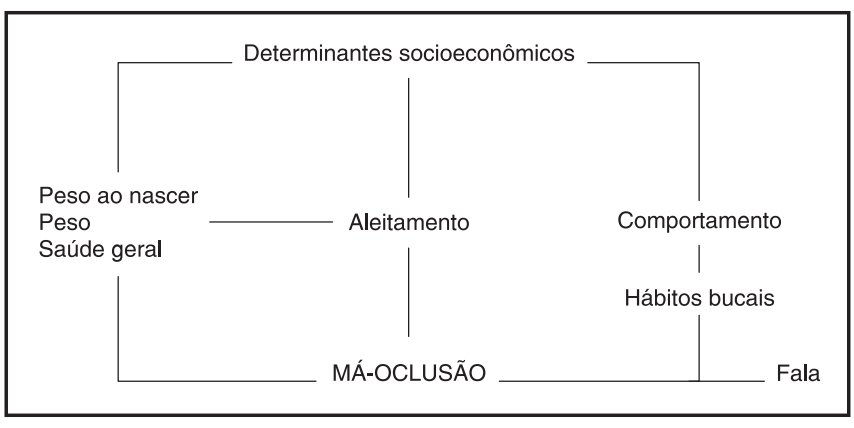

FIGURA 1 - Determinantes socioeconômicos da máoclusão - modelo teórico.

amostra. Foi realizada a amostragem por conglomerado, com escolha aleatória de 30 pré-escolas, representando $30 \%$ de cada categoria de instituição.

Para determinar a subamostra, foi realizado sorteio de 50,0\% das crianças efetivamente examinadas. Foi solicitado aos pais/responsáveis das mesmas que respondessem ao questionário socioeconômico, com 54,5\% de taxa de respostas.

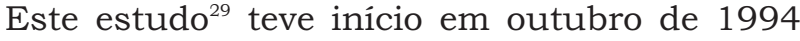
com delineamento do tipo transversal e foi concluído em novembro de 1995. Foi composto por três etapas: exame de oclusão, medidas antropométricas e questionário socioeconômico. Uma amostra de 2.139 crianças de 3 a 5 anos foi avaliada para o exame de oclusão e medidas antropométricas e uma subamostra de 618 crianças apresentou resposta ao questionário socioeconômico. As respostas dos pais desta subamostra de crianças ao questionário serão alvo de discussão no presente artigo.

O questionário aplicado aos pais consistiu de questões fechadas e abertas, contemplando assuntos como identificação, composição familiar, escolaridade paterna e materna, renda familiar, condição de emprego, ocupação da pessoa de maior renda, trabalho da mãe, condições de moradia e saneamento e posse de bens. Adicionalmente, condições relativas à saúde da criança foram avaliadas por intermédio de questões sobre consultas médicas, presença de problemas respiratórios, cuidados com a saúde bucal, visita ao cirurgião-dentista e motivo desta visita e aleitamento materno.

Algumas variáveis consideradas "ambientais" na etiologia das más-oclusões foram abordadas no questionário, como distúrbios de fala e presença de hábitos bucais (dormir com objetos na boca, dormir sobre as mãos, dormir de um só lado, respiração bucal, morder objetos/unhas/lábios, bruxismo, sucção de chupeta, sucção digital, interposição de língua e deglutição atípica). 
QUADRO 1 - Principais estudos sobre hábitos bucais e má-oclusão em pré-escolares.

\begin{tabular}{|c|c|c|c|c|c|c|c|c|c|c|c|c|c|}
\hline Autor & País & Ano & $\begin{array}{l}\text { Delinea- } \\
\text { mento }\end{array}$ & Amostra & $\mathrm{N}$ & $\begin{array}{l}\text { Idade } \\
\text { (anos) }\end{array}$ & $\begin{array}{l}\text { Classifi- } \\
\text { cação }\end{array}$ & $\begin{array}{l}\text { Método de } \\
\text { análise }\end{array}$ & Hábito & $\%$ & Sexo & Variáveis & Resultados \\
\hline $\begin{array}{l}\text { NANDA } \\
\text { et } a l^{20}\end{array}$ & Índia & 1972 & transversal & - & 2.500 & $2-6$ & $\begin{array}{l}\text { Foster; } \\
\text { Hamilton }\end{array}$ & - & polegar & 17,0 & $\mathrm{~F}$ & + insegurança & $\begin{array}{l}\text { “overjet” } \\
\text { Classe II }\end{array}$ \\
\hline $\begin{array}{l}\text { POPOVICH; } \\
\text { THOMPSOM }^{23}\end{array}$ & Canadá & 1973 & longitudinal & - & 1.258 & $3-12$ & Cl. I, II, III & bivariada & digital & 30,0 & - & - pacificador & Classe II \\
\hline INFANTE $^{13}$ & USA & 1976 & transversal & aleatória & 680 & $2,5-6$ & $\begin{array}{l}\text { Foster; } \\
\text { Hamilton }\end{array}$ & bivariada & digital & 18,7 & $\mathrm{~F}$ & - nível s. ec. & $\begin{array}{l}\text { m. cruzada* } \\
\text { Classe II }\end{array}$ \\
\hline $\begin{array}{l}\text { KISLING; } \\
\text { KREBS }^{15}\end{array}$ & Dinamarca & 1976 & - & - & 1.624 & 3 & $\begin{array}{l}\text { R. molar } \\
\text { canina }\end{array}$ & bivariada & chupeta & 26,0 & - & - & m. cruzada \\
\hline ZADIK et $a l .^{30}$ & Israel & 1977 & transversal & - & 333 & $0-7$ & - & bivariada & $\begin{array}{l}\text { digital } \\
\text { chupeta }\end{array}$ & $\begin{array}{l}95,0 \\
69,0\end{array}$ & - & + indulgência & - \\
\hline CERNY $^{5}$ & Austrália & 1981 & transversal & - & 600 & - & - & - & $\begin{array}{l}\text { digital } \\
\text { chupeta }\end{array}$ & 18,0 & $\mathrm{~F}$ & - & $\begin{array}{l}\text { distorções } \\
\text { arco }\end{array}$ \\
\hline OLIVEIRA $^{22}$ & Brasil & 1981 & transversal & - & 790 & $3-5$ & Baume & bivariada & chupeta & - & $\mathrm{F}$ & - idade & m. aberta** \\
\hline DE VIS et $a l .{ }^{9}$ & Bélgica & 1984 & transversal & randômica & 510 & $3-6$ & - & bivariada & chupeta & 45,1 & - & $\begin{array}{l}\text { chupeta } \\
>\text { digital }\end{array}$ & m. aberta \\
\hline $\mathrm{SCHLOMER}^{24}$ & Alemanha & 1984 & - & - & 582 & $3-6$ & - & - & $\begin{array}{l}\text { polegar } \\
\text { chupeta }\end{array}$ & - & - & - & $\begin{array}{l}\text { m. cruzada } \\
\text { m. aberta }\end{array}$ \\
\hline $\begin{array}{l}\text { CASTELANI } \\
\text { et al. }{ }^{4}\end{array}$ & Itália & 1987 & transversal & aleatória & 400 & 3 e 5 & Angle & - & chupeta & 67,8 & - & $\begin{array}{l}\text { predisposição } \\
\text { hereditária }\end{array}$ & $\begin{array}{l}\text { m. aberta } \\
\text { Classe II }\end{array}$ \\
\hline KEROSUO $^{14}$ & $\begin{array}{l}\text { Tanzânia } \\
\text { Finlândia }\end{array}$ & 1989 & transversal & - & $\begin{array}{l}580 \\
575\end{array}$ & $3-8$ & R. sagital & $\begin{array}{l}\text { bivariada } \\
\text { multivariada }\end{array}$ & digital & $\begin{array}{l}14,0 \\
10,0\end{array}$ & - & - & $\begin{array}{l}\text { m. aberta } \\
\text { m. cruzada }\end{array}$ \\
\hline $\mathrm{ADAIR}^{1}$ & USA & 1992 & transversal & - & 79 & $2-5$ & $\begin{array}{l}\text { R. molar } \\
\text { canina }\end{array}$ & bivariada & chupeta & - & M & + horas/dia & Classe II \\
\hline $\begin{array}{l}\text { OGAARD } \\
\text { et } \text { al. }^{21}\end{array}$ & $\begin{array}{l}\text { Suécia } \\
\text { Noruega }\end{array}$ & 1994 & transversal & - & 445 & 3 & - & multivariada & $\begin{array}{l}\text { digital } \\
\text { chupeta }\end{array}$ & - & $\mathrm{F}$ & + anos uso & m. cruzada \\
\hline
\end{tabular}

* Mordida cruzada posterior; ** Mordida aberta anterior.

Fonte: TOMITA, N. E. Relação entre determinantes socioeconômicos e hábitos bucais: influência na oclusão de pré-escolares de Bauru - SP Brasil. Bauru, 1996. 246 p. Tese (Doutorado) - Faculdade de Odontologia de Bauru, Universidade de São Paulo. 
TOMITA, N. E.; SHEIHAM, A.; BIJELLA, V. T.; FRANCO, L. J. Relação entre determinantes socioeconômicos e hábitos bucais de risco para más-oclusões em pré-escolares. Pesq Odont Bras, v. 14, n. 2, p. 169-175, abr./jun. 2000.

Na totalização dos resultados, foram computadas apenas as respostas válidas, ou seja, respostas completas às perguntas formuladas. Em análise preliminar das informações coletadas, foi possivel verificar que algumas variáveis associadas à má-oclusão na literatura especializada apresentaram freqüência muito reduzida nesta população. Assim, apenas os hábitos bucais sucção de chupeta e sucção digital constituirão o alvo da presente análise como variáveis dependentes.

Os dados foram processados através do software Epi Info Versão $5.01 \mathrm{~b}^{10}$. Foram utilizados o teste do qui-quadrado e teste de Fisher ${ }^{26}$ para a análise bivariada envolvendo as respostas ao questionário socioeconômico e algumas variáveis de exposição. Fixou-se em 0,05 o nivel para rejeição da hipótese de nulidade, assinalando-se com um asterisco os valores significantes na apresentação das tabelas.

\section{RESULTADOS \\ Representatividade da subamostra}

Na Tabela 1, verificam-se algumas características da composição dos grupos que demonstram a representatividade da subamostra.

\section{Renda familiar}

A renda familiar total foi estratificada em cinco categorias (até $R \$ 200, R \$ 201-500, R \$ 501-1.000$, $\mathrm{R} \$$ 1.001-1.500 e > R\$ 1.500) e avaliada quanto à sua associação com os principais hábitos bucais: sucção de chupeta e sucção digital.

O hábito de sucção de chupeta apresentou tendência a ser mais freqüente entre as crianças de renda familiar mais baixa, para o sexo masculino e feminino, decrescendo com o incremento da renda. As diferenças não apresentaram significância

TABELA 1 - Distribuição de crianças da amostra e subamostra, segundo idade e sexo. Bauru - SP, 1995.

\begin{tabular}{c|r|c|c|c|c|c|c|c}
\hline \hline \multirow{2}{*}{$\begin{array}{c}\text { Idade } \\
\text { (anos) }\end{array}$} & \multicolumn{4}{|c|}{ Amostra } & \multicolumn{4}{c}{ Subamostra } \\
\cline { 2 - 9 } & \multicolumn{1}{|c|}{ Masculino } & \multicolumn{1}{c|}{ Feminino } & \multicolumn{2}{c}{ Masculino } & \multicolumn{2}{c}{ Feminino } \\
\hline 3 & 240 & 21,2 & 216 & 21,5 & 80 & 25,8 & 73 & 23,7 \\
\hline 4 & 407 & 35,9 & 367 & 36,5 & 109 & 35,2 & 111 & 36,0 \\
\hline 5 & 487 & 42,9 & 422 & 42,0 & 121 & 39,0 & 124 & 40,3 \\
\hline Total & 1134 & 100,0 & 1005 & 100,0 & 310 & 100,0 & 308 & 100,0 \\
\hline \hline
\end{tabular}

Amostra: $X^{2}=0,20 ; X^{2}$ crítico $=5,99$

Subamostra: $X^{2}=0,37 ; X^{2}$ crítico $=5,99$. estatística. A freqüência de sucção digital entre as crianças de ambos os sexos foi bastante baixa, sem influência do estrato de renda familiar.

\section{Escolaridade materna}

As respostas à questão escolaridade materna foram categorizadas $\mathrm{em}: 1^{\circ}$ grau incompleto, $2^{\circ}$ grau incompleto ou completo e universitário.

A freqüência de crianças que apresentam o hábito de sucção de chupeta apresentou tendência decrescente com o incremento da escolaridade materna, para o sexo masculino e para o feminino. Em ambos os casos, as diferenças não tiveram significância estatística.

O hábito de sucção digital não apresentou relação com o nível de escolaridade das mães entrevistadas. A freqüência de crianças com o hábito de sucção digital não apresentou diferenças estatisticamente entre os grupos, em ambos os sexos.

\section{Trabalho materno}

As respostas à questão se as mães trabalharam nos 12 meses anteriores ao questionário foram agrupadas em três categorias - sim, não ou outra situação. Esta última abrangeu a condição de estudante, trabalho eventual ou trabalho remunerado, realizado em casa.

O uso de chupeta não diferiu significantemente entre grupos, para o sexo masculino. Para o sexo feminino, no entanto, a freqüência de crianças com este hábito foi significantemente maior para aquelas cujas mães estão inseridas no mercado de trabalho (Tabela 2).

Apenas 4,9\% dos meninos e $6,7 \%$ das meninas apresentavam o hábito de sucção digital, que não apresentou diferenças estatisticamente significan-

TABELA 2 - Prevalência de sucção de chupeta entre as crianças da subamostra, segundo o trabalho materno e sexo. Bauru - SP, 1995.

\begin{tabular}{l|c|c|c|c}
\hline \hline \multirow{2}{*}{$\begin{array}{c}\text { Trabalho } \\
\text { materno }\end{array}$} & \multicolumn{4}{|c}{ Sucção de chupeta } \\
\cline { 2 - 5 } & \multicolumn{2}{|c|}{ Masculino } & \multicolumn{2}{c}{ Feminino } \\
\cline { 2 - 5 } & $\mathrm{N}$ & $\%$ & $\mathrm{~N}$ & $\%$ \\
\hline Não (A) & $38 / 150$ & 29,2 & $34 / 124$ & 27,4 \\
\hline Sim (B) & $54 / 163$ & 33,1 & $61 / 158$ & 38,6 \\
\hline Outra situação (C) & $3 / 12$ & 25,0 & $4 / 22$ & 18,2 \\
\hline Total & $95 / 305$ & 31,2 & $99 / 304$ & 32,6 \\
\hline \hline
\end{tabular}

Masculino: $X^{2}=0,73 ; X^{2}$ crítico $=5,99$;

Feminino: $X^{2}=6,20^{*} ; X^{2}$ crítico $=5,99 ; B>(A+C)^{*}$. 
TOMITA, N. E.; SHEIHAM, A.; BIJELLA, V. T.; FRANCO, L. J. Relação entre determinantes socioeconômicos e hábitos bucais de risco para más-oclusões em pré-escolares. Pesq Odont Bras, v. 14, n. 2, p. 169-175, abr./jun. 2000.

tes na distribuição entre as crianças de ambos os sexos, segundo a variável trabalho materno.

\section{Ocupação dos pais}

A ocupação da pessoa que contribui mais efetivamente no orçamento familiar foi classificada segundo oito categorias: proprietários; administradores, gerentes, diretores; profissionais de nivel superior; funções de escritório; trabalhadores manuais especializados; trabalhadores manuais semi-especializados; trabalhadores manuais nãoqualificados; fora da população economicamente ativa $^{9}$. Estas categorias foram agrupadas, resultando em três categorias finais: especializada (que englobou as cinco primeiras categorias); semiespecializada (equivalente a trabalhadores manuais semi-especializados); manual (equivalente a trabalhadores manuais não-qualificados). As respostas "fora da população economicamente ativa" não foram consideradas nesta análise.

O hábito de sucção de chupeta apresentou freqüência significantemente menor para os meninos na categoria ocupação paterna especializada que nas categorias semi-especializada e manual, que não diferiram entre si. Para as meninas, houve uma tendência a menor freqüência deste hábito bucal na categoria ocupação paterna especializada (Tabela 3).

O hábito de sucção digital, por sua vez, não apresentou diferenças estatisticamente significantes na distribuição de casos entre as categorias, em ambos os sexos.

\section{DISCUSSÃO}

"A sustentação de uma relação causal entre duas variáveis complexas não é tarefa para apenas

TABELA 3 - Prevalência de sucção de chupeta entre as crianças da subamostra, segundo a ocupação da pessoa de maior renda no domicílio e sexo. Bauru - SP, 1995.

\begin{tabular}{l|l|c|c|c}
\hline \hline \multirow{2}{*}{ Ocupação } & \multicolumn{3}{c}{ Sucção de chupeta } \\
\cline { 2 - 5 } & \multicolumn{2}{|c|}{ Masculino } & \multicolumn{2}{c}{ Feminino } \\
\cline { 2 - 5 } & $\mathrm{N}$ & $\%$ & $\mathrm{~N}$ & $\%$ \\
\hline Especializada (A) & $24 / 116$ & 20,7 & $32 / 129$ & 24,8 \\
\hline Semi-especial (B) & $44 / 115$ & 38,3 & $40 / 104$ & 38,5 \\
\hline Manual (C) & $22 / 56$ & 39,3 & $22 / 58$ & 37,9 \\
\hline Total & $90 / 287$ & 31,4 & $94 / 291$ & 32,3 \\
\hline \hline
\end{tabular}

Masculino: $\mathrm{X}^{2}=10,31^{*} ; \mathrm{X}^{2}$ crítico $=5,99 ; \mathrm{A}<(\mathrm{B}+\mathrm{C})^{*}$

Feminino: $X^{2}=5,96 ; X^{2}$ crítico $=5,99$. um estudo. Ao contrário, depende da sistematização dos resultados de muitos estudos epidemiológicos e não-epidemiológicos. Entretanto, se as evidências de um estudo forem avaliadas criteriosamente, poderão apoiar uma interpretação causal de seus achados"11. As evidências de uma associação significativa $(\mathrm{p}<0,05)$ entre alguns determinantes socioeconômicos e as variáveis ambientais (hábitos bucais) e destas, por sua vez, com a prevalência de má-oclusão, reforçam a suposição de uma relação causal.

A identificação das variáveis trabalho materno e ocupação dos pais como determinantes de hábitos de sucção de chupeta e sucção digital concordou com a hipótese teórica. No entanto, discordou de estudos disponíveis ${ }^{3,9}$, que não reportaram associações entre variáveis socioeconômicas e hábitos bucais. Alguns estudos abordam aspectos de ordem psicológica como determinantes de hábitos bucais ${ }^{16,17,18,20}$, relegando os determinantes sociais, econômicos e demográficos a um segundo plano.

Parece evidente que o trabalho remunerado das mães as distancia de seus filhos, que, de alguma maneira, buscam uma compensação emocional que se traduz na continuidade de hábitos bucais após a idade de três anos. A ocupação manual (da pessoa de maior renda no domicílio) predispôs à maior freqüência de crianças com hábitos, o que indica que a falta de informações e baixo acesso a cuidados de saúde apresentados por este grupo podem repercutir negativamente no desenvolvimento infantil. Assim, determinantes socioeconômicos afirmam-se como variáveis causais em relação aos hábitos bucais. Estes afirmam-se como variáveis independentes ou causais em relação à má-oclusão, tanto em estudos epidemioló$\operatorname{gicos}^{1,4,5,9,13,14,15,20,21,22,23,24,29,30}$ como não-epidemioló$\operatorname{gicos}^{16,17,18,27,28}$.

Embora se reconheça a importância dos determinantes socioeconômicos sobre a saúde infanti $1^{8,11,19}$, este estudo possibilitou compreendê-la no campo da saúde bucal.

É provável que o cotidiano do cuidado infantil se apresente como um reflexo da inserção das mães no mercado de trabalho. Ficou evidente que a resposta da criança frente ao distanciamento da mãe, é manifestar, através de hábitos regredidos, mecanismos de compensação às sensações de insegurança.

A ocupação da pessoa de maior renda no domicílio também se destaca por sua associação com os hábitos bucais. Esta variável apresenta relação di- 
TOMITA, N. E.; SHEIHAM, A.; BIJELLA, V. T.; FRANCO, L. J. Relação entre determinantes socioeconômicos e hábitos bucais de risco para más-oclusões em pré-escolares. Pesq Odont Bras, v. 14, n. 2, p. 169-175, abr./jun. 2000.

reta com as variáveis escolaridade e renda, que decrescem do nível especializado para o nível manual de ocupação. Entre as ocupações de caráter manual, verificou-se maior prevalência de hábitos, sendo correto conjecturar que a baixa escolaridade e acesso reduzido a informações possa levar os pais a reproduzir práticas de cuidado infantil, como o uso continuado de chupeta. Ou seja, mesmo cessado o período do desenvolvimento infantil em que os hábitos bucais são considerados fisiológicos, sua interrupção parece não ser preocupação dos pais, que se defrontam com as dificuldades do mercado de trabalho e o cotidiano da subsistência.

É necessário desvendar os complexos mecanismos que interagem simultaneamente e propiciam às variáveis socioeconômicas determinar efeitos sobre a ocorrência de más-oclusões, através da permanência de hábitos bucais em idade pré-escolar, e futuros estudos com delineamento longitudinal podem oferecer contribuições neste sentido.

\section{CONCLUSÕES}

1. Alguns determinantes socioeconômicos, como o trabalho materno e ocupação da pessoa de maior renda no domicílio estão associados com a maior prevalência de sucção de chupeta em pré-escolares.

2. As variáveis renda familiar e escolaridade materna não apresentaram associação com a presença de hábitos bucais nesta população.

\section{AGRADECIMENTOS}

Aos Profs. Drs. Eymar Sampaio Lopes, José Alberto de Souza Freitas, José Mondelli, Luiz Reynaldo de Figueiredo Walter, Neil Ferreira Novo, Omar Gabriel da Silva Filho, Paulo Nadanovsky, Ricardo Cordeiro e Yara Juliano; aos Cirurgiões-Dentistas Ana Maria Campos, Ana Virgínia Sampaio, César de Oliveira, Claudia de Morais, Dayseane Silva, Émerson Gazzoli, Lindaura Monteiro, Luciene Rodrigues (in memorian), Maurício Pinheiro, Patrícia Teixeira, Renata Pernambuco, Renata Mantovani e Teresa Lima Baptista; às THDs Marta Liporacci Aoki e Helena Mantovani, nossos agradecimentos.

TOMITA, N. E.; SHEIHAM, A.; BIJELLA, V. T.; FRANCO, L. J. The relationship between socioeconomic determinants and oral habits as risk factors for malocclusion in preschool children. Pesq Odont Bras, v. 14, n. 2, p. 169-175, abr./jun. 2000 .

In order to evaluate how socioeconomic determinants affect the prevalence of oral habits in preschool children, this cross-sectional study was developed. The survey was carried out from October, 1994 to December, 1995. A random sample of 2,139 children aged 3 to 5 years old was evaluated. The children were enrolled in private or public institutions in the Municipal District of Bauru, São Paulo, Brazil. A sub-sample of 618 children presented response to the socioeconomic questionnaire. The considered hypothesis is that socioeconomic determinants affect the psychological status of the child, and it is observed through the development of deleterious oral habits, like dummy-sucking or digit-sucking. The results were tested by bivariate analysis (chi-square test). Some social determinants, like the mother's employment and the occupation of the person who has the greater income in the household, are related to oral habits $(\mathrm{p}<0.05)$, which in turn are strongly associated with malocclusion.

UNITERMS: Epidemiology; Malocclusion; Socioeconomic factors; Oral habits.

\section{REFERÊNCIAS BIBLIOGRÁFICAS}

1. ADAIR, S. M.; MILANO, M.; DUSHKU, J. C. Evaluation of the effects of orthodontic pacifiers on the primary dentitions of 24- to 59-month-old children: preliminary study. Pediatr Dent, v. 14, n. 1, p. 13-18, Jan./Feb. 1992.

2. BARROS, F. C.; VICTORA, C. G. Epidemiologia da saúde infantil: um manual para diagnósticos comunitários. São Paulo : HUCITEC/UNICEF, 1991.

3. CALISTI, L.; COHEN, M. M.; FALES, M. H. Correlation between malocclusion, oral habits, and socio-economic levels of preschool children. J Dent Res, v. 39, n. 3, p. 450-454, May/June 1960.

4. CASTELLANI, G.; BERTELE, G. P.; ZERMAN, N. Indagine epidemiologica nelle scuole materne del Comune di Ve- rona sullincidenza della carie, delle malocclusioni e delle abitudini viziate che possono influire sul normale sviluppo delle strutture scheletriche facciali del bambino. Minerva Stomatol, v. 36, n. 3, p. 121-125, Mar. 1987.

5. CERNY, R. Thumb and finger sucking. Aust Dent J, v. 26, n. 3, p. 167-171, June 1981.

6. CHAVES, M. M. Problemas. In:_ Odontologia social. 3. ed. Rio de Janeiro : Artes Médicas, 1986. Cap. 2, p. 23-98.

7. COCHRAN, W. G. Sampling techniques. New York : John Wiley \& Sons, 1977.

8. CORREIA, L. L.; MCAULIFFE, J. F. Saúde materno-infantil. In: ROUQUAYROL, M. Z. Epidemiologia e saúde. 4. ed. Rio de Janeiro : MEDSI, 1994. Cap. 11, p. 315-342. 
TOMITA, N. E.; SHEIHAM, A.; BIJELLA, V. T.; FRANCO, L. J. Relação entre determinantes socioeconômicos e hábitos bucais de risco para más-oclusões em pré-escolares. Pesq Odont Bras, v. 14, n. 2, p. 169-175, abr./jun. 2000.

9. DE VIS, H.; DE BOEVER, J. A.; VAN CAUWENBERGHE, P. Epidemiologic survey of functional conditions of the masticatory system in Belgian children aged 3-6 years. Community Dent Oral Epidemiol, v. 12, n. 3, p. 203-207, June 1984.

10. DEAN A. G.; DEAN, J. A.; BURTON, A. H. et al. Epi Info, Version 5: a word processing, database, and statistics program for epidemiology on micro-computers. [computer program]. Atlanta : Centers for Disease Control, 1990.

11. FACCHINI, L. A. Trabalho materno e ganho de peso infantil. Pelotas : UFPel, 1995.

12. FREITAS J. A. de S.; LOPES, E. S.; ALVARES, L. C. et al. Variabilidade das fases de formação e erupção dos dentes permanentes. Ortodontia, v. 23, n. 2, p. 29-38, 1990.

13. INFANTE, P. F. An epidemiologic study of finger habits in preschool children as related to malocclusion, socioeconomic status, race, sex and size of community. J Dent Child, v. 43, p. 33-38, Jan./Feb. 1976.

14. KEROSUO, H. Occlusion in the primary and early mixed dentitions in a group of Tanzanian and Finnish children. J Dent Child, v. 57, n. 4, p. 293-298, July/Aug. 1990.

15. KISLING, E.; KREBS, G. Patterns of occlusion in 3-year-old Dannish children. Community Dent Oral Epidemiol, v. 4, p. 152-159, 1976.

16. KLEIN, E. T. The thumb-sucking habit: meaningful or empty? Am J Orthodont, v. 59, n. 3, p. 283-289, Mar. 1971.

17. MARTINEZ, N. P.; HUNCKLER Jr., R. J. Managing digital habits in children. Comp Contin Educ Dent, v. 6, n. 3, p. 188-197, Mar. 1985.

18. MASSLER, M. Oral habits: development and management. J Pedod, v. 7, n. 2, p.109-119, Winter 1983.

19. MONTEIRO, C. A. Saúde e nutrição das crianças de São Paulo. São Paulo : HUCITEC/EDUSP, 1988.

20. NANDA, R. S.; KHAN, I.; ANAND, R. Effect of oral habits on the occlusion in preschool children. J Dent Child, v. 39, p. 449-452, Nov./Dec. 1972.

21. OGAARD, B.; LARSSON, E.; LINDSTEN, R. The effect of sucking habits, cohort, sex, intercanine arch widths, and breast or bottle feeding on posterior crossbite in Norwegian and Swedish 3-year-old children. Am J Orthod Dentofacial Orthop, v. 106, n. 2, p. 161-166, Aug. 1994.

22. OLIVEIRA, S. F. Oclusão e hábitos de sucção: estudo em pré-escolares de Piracicaba. Piracicaba, 1981. 61 p. Dissertação (Mestrado) - Faculdade de Odontologia de Piracicaba, Universidade Estadual de Campinas.

23. POPOVICH, F.; THOMPSON, G. W. Thumb and finger-sucking: its relation to malocclusion. Am J Orthodont, v. 63, n. 2, p. 148-155, Feb. 1973.

24. SCHLOMER, R. Influence of thumb sucking and pacifiers on deciduous teeth. Fortschr Kieferorthop, v. 45, n. 2, p. 141-148, Apr. 1984. /Abstract/

25. SCHNEIDER, P. E.; PETERSON, J. Oral habits: considerations in management. Pediatr Clin North Am, v. 29, n. 3, p. 523-546, June 1982.

26. SIEGEL, S. Estadística no parametrica. Mexico : Ed. Trillas, 1975.

27. SILVA FILHO, O. G. da; OKADA, T.; SANTOS, S. D. dos. Sucção digital: abordagem multi-disciplinar: Ortodontia x Psicologia x Fonoaudiologia. Estomat Cult, v. 16, n. 2, p. 44-52, 1986.

28. SUBTELNY, J. D.; SUBTELNY, J. D. Oral habits - studies in form, function and therapy. Angle Orthod, v. 43, n. 4 , p. 347-383, Oct. 1973.

29. TOMITA, N. E. Relação entre determinantes socioeconômicos e hábitos bucais: influência na oclusão de pré-escolares de Bauru - SP - Brasil. Bauru, 1996. 246 p. Tese (Doutorado) - Faculdade de Odontologia de Bauru, Universidade de São Paulo.

30. ZADIK, D.; STERN, N.; LITNER, M. Thumb and pacifier sucking habits. Amer J Orthod, v. 71, n. 3, p. 197-201, Feb. 1977.
Recebido para publicação em 13/01/00

Enviado para reformulação em 14/03/00

Aceito para publicação em 28/04/00 